\title{
Optimal Design on Fitness Equipment Distribution Route
}

\author{
Yanjie Li \\ Department of Physical Education \\ Bohai University \\ Jinzhou, P.R. China \\ 747228464@qq.com
}

\author{
Wenwei Jia \\ Department of Physical Education \\ Bohai University \\ Jinzhou, P.R. China \\ 894161757@qq.com
}

\begin{abstract}
With the progress of society, the continuous improvement of living standards, people are increasingly strong demand for fitness. Fitness equipment plays a very important role in the national fitness. Therefore, it has important significance to the reasonable distribution route for fitness equipment business. This paper studies the design of fitness equipment distribution route optimization. First of all, introduces two more mature issues in distribution optimization model: TSP and VRP problem; then, describes the method to solve the VRP problem: saving algorithm, expounds its basic ideas. Finally, the design optimization model of distribution lines, and introduces the application of fitness equipment distribution lines in solving steps. Not only can shorten the transport distance, saving cost and time, but also is an important way for enterprises to improve service quality, enhance competitiveness.
\end{abstract}

Keywords-distribution route; fitness equipment; VRP; saving algorithm

\section{INTRODUCTION}

With the rapid development of social productive forces, the continuous improvement of people's living standards, more and more people began to pay attention to their physical and mental health. People need to fitness equipment is also increasing, as major units, schools, fitness equipment ordered is more. Therefore, for the fitness equipment business, reasonable distribution route is essential. Distribution path optimization problem is always an important problem in the study of logistics. The distribution route is refers to various delivery vehicles to each user delivery to go through the line, distribution lines is reasonable or not has a direct impact on the rational use of vehicles and the distribution costs [1]. Rational distribution path design, not only can shorten the transport distance, cost and time savings, which directly reduce costs, but also an important way for enterprises to improve service quality, enhance competitiveness. For businesses, in the face of tough competition in the market at the same time, a reasonable set of logistics distribution routing optimization, it is sufficient to highlight their own logistics service characteristics, is an important choice. In short, the distribution route optimization is a major problem in the distribution work, related to the sustainable development of enterprises and society, has important theoretical significance and application value [2]. Therefore, the combination of logistics and distribution shortest path optimization, and explore fitness equipment business practical logistics distribution routing optimization options, become the main purpose of this article. With the complex of the distribution, optimized distribution lines are generally combined with mathematical methods and computer solving methods to develop a reasonable distribution program.

Vehicle route problem is one of the well-known NP-hard combinatorial optimization field, before computers and other modern information technology does not appear, the process of solving this problem is quite difficult and complex, often require application-related technology issues decomposed or converted into one or more has been studied the basic question before, such as the traveling salesman problem, the shortest path problem, the shortest flow problem, the minimum cost maximum flow problem, postman problems, and so on. Then use more mature theories and methods to solve, in order to achieve the best original vehicle route problem solution or satisfactory solution. Currently, in the aspect of distribution optimization model, mainly are the traveling salesman problem and vehicle routing problem of two kinds of comparatively mature. Traveling salesman problem (TSP) refers to a distribution center from there shipped to the customer, the center according to customer' requirements, determine the route, and vehicle scheduling. At the same time, given the quantity of each customer does not exceed the capacity of the car, if more, the first take a direct distribution way to send a certain number of goods, the remaining dissatisfied vehicle goods then touring vehicle distribution. Vehicle routing problem (VRP) is an important expansion and extension of the TSP problem. It is with a capacity constraint on a group of team (the number of vehicles, capacity, speed) of scheduling and routing, which in one or several distribution outlets distribution of goods to customers, and meet the needs of the customer orders, ultimately determine that these vehicles complete the allocation of tasks to go through the line, so that the final cost is the lowest [3].

\section{SAVING AlgORITHM}

Distribution vehicle route arrangement problem can be described as: transport vehicles from one or more facilities to multiple geographically dispersed customer sites, design optimization of a flow of goods transport routes, while meeting a series of constraints. The premise condition of the problem is facility location, customer location and road condition is known, which can determine a vehicle transport routes, in order to meet the objective function [4]. Solve this problem, there are two types of algorithms, respectively is: 
exact algorithms and heuristics algorithms. Exact algorithms generally apply only to small scale vehicle routing problem solving, because the algorithm is based on a rigorous mathematical methods, in the process of solving often requires a lot of mathematical calculations [5]. Heuristics algorithms are within acceptable time limits under the optimal solution of the problem as much as possible, such as tabu search algorithm, scanning method, nearest neighbor or interpolation method, ant colony algorithm, these algorithms for solving the VRP problem provides an effective method, can be a viable solution in a short time.

When the problem by a simple linear problem into a nonlinear problem, the complexity of the problem increases, and the size of the problem becomes large, then the use of precise algorithm to solve becomes infeasible, the optimal solution cannot be solved within the effective time. And in this case, is usually applied heuristic algorithm to solve the problem, get "near optimal solution" or "satisfactory solution", the algorithm is simple and convenient, easy to implement [6]. A more mature approach to optimize the distribution scheme: $\mathrm{C}-\mathrm{W}$ saving method, also called saving mileage algorithm. Saving algorithm is proposed by Clark and Wright, one of the most classic construction heuristics in 1964. The main idea of the algorithm is: from not on the line of the customer node, located between each customer nodes through a connection point can save a maximum distance (saving value), which is inserted into the current route; gradually repeat the operation until all points in the distribution route.

The basic idea of saving algorithm is [7]: the set $P_{0}$ point for the distribution center location, $P_{1}, P_{2}, \cdots, P_{m}$ for $m$ customer's location, the shortest distance is $d_{i, j}$ between $P_{i}$ and $P_{j}$, and $d_{i, j}$ is known $(i, j=1,2,3, \ldots, m)$, between distribution centers and customers the shortest distance is $d_{0, m}$, and $d_{0, m}$ is $\operatorname{known}(m=1,2,3, \ldots) \cdot d_{0, i}$ represents the distance from the distribution centers $o$ to customers $i$, $d_{0, j}$ represents the distance from the distribution center $o$ to the customer $j, d_{i, j}$ represents the distance from the customer $i$ to the customer $j$, as shown in Figure 1.

Scheme of $a$, the distribution route:

$$
\begin{aligned}
& p_{0} \rightarrow p_{\mathrm{i}-1} \rightarrow p_{\mathrm{i}} \rightarrow p_{0} \\
& p_{0} \rightarrow p_{\mathrm{j}+1} \rightarrow p_{\mathrm{j}} \rightarrow p_{0} \\
& \text { The total distribution distance: } \\
& \qquad D_{1}=\left(d_{0, i-1}+d_{i-1, i}+d_{0, i}\right)+\left(d_{0, j}+d_{j, j+1}+d_{0, j+1}\right)
\end{aligned}
$$

Scheme of $b$, the distribution route:

$$
p_{0} \rightarrow p_{\mathrm{i}-1} \rightarrow p_{\mathrm{i}} \rightarrow p_{j} \rightarrow p_{j+1} \rightarrow p_{0}
$$

The total distribution distance:

$$
D_{1}=d_{0, i-1}+d_{i-1, i}+d_{i, j}+d_{j, j+1}+d_{0, j+1}
$$

For both schemes, the smaller the distribution distance, so the more reasonable the scheme, formula (1) minus the formula (2) to get:

$$
s_{i, j}=D_{1}-D_{2}=d_{0, i}+d_{0, j}+d_{i, j}
$$

The principle of saving algorithm is: any two sides of a triangle are greater than the third side, to Fig. 1, even $P_{0}, P_{i}$, $P_{j}$ as a triangle:

$$
s_{i, j}=D_{1}-D_{2}=d_{0, i}+d_{0, j}+d_{i, j}>0
$$

so $D_{1}>D_{2}$.

By formula (4) shows the scheme $b$ is superior to the scheme $a$.

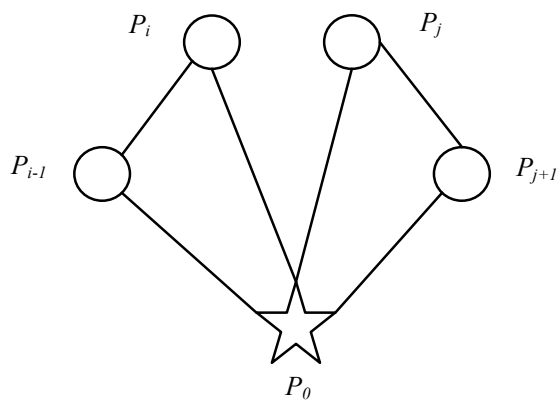

(a)

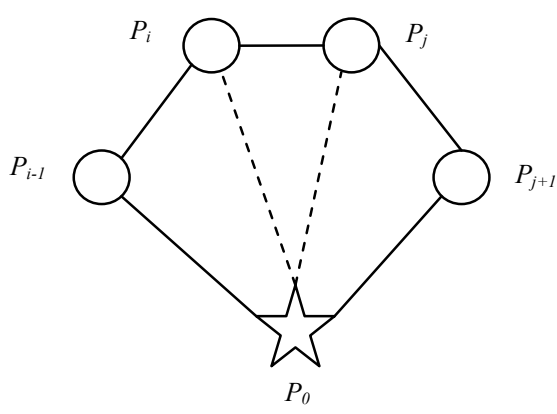

(b)

Figure 1. Route scheme diagram

$s_{i, j}$ is greater, indicating when the total distance and connected together to reduce more. In order to facilitate the analysis, this saves distance defined as "savings value", formula (4) represent.

Based on the basic idea of saving mileage method, to find such a triangular loop in the distribution network: as far as possible loading of goods, and save as much as possible mileage. Saving algorithm to solve the VRP steps are: enterprise distribution center as a starting point, connecting the start point to the end of each client for separation route begins; use equation (4) to calculate mileage savings; all the saving value is placed in a set, sort from largest to smallest savings value; If set equal to null, then the algorithm stops; otherwise, the first item savings value in the set, if meet the condition connecting the two points, these conditions are: two points are not in the optimization circuit; two points in the route, but not within the line points; two points in optimizing the circuit, but the one is the starting point the other one is the end point. according to the saving value connected clients point forming route. Be careful not to damage any lines previously connected, when all clients connected to the route, and distribution lines to complete [8]. 


\section{Distribution ROUTE MODEL}

Assumption: a distribution center to provide distribution services to its target customers, P0 expressed as a distribution center, the customer is expressed as $\mathrm{Pi}$, where $(i=1,2,3, \ldots, m) \cdot d_{i, j}$ represents the distance from the customer $i$ to customer $j, d_{0, j}$ expressed the distance from the distribution center $o$ to the customer i. Each distance corresponds to a certain costs, $c_{i, j}(i, j=1,2,3 \ldots, m)$ said the cost of the distribution center to the customer or between each customer point. Distribution center vehicle number $k$, rated load $q$, the vehicle must not be overloaded $g_{i}(i=1,2,3 \ldots, m)$. is the demand of $i$-ed customer.

In order to establish the model, the binary variable is defined as follows [9]:

$x_{i j k}=1$ represents $k$ vehicle from client $i$ to $j$; otherwise $x_{i j k}=0$;

$y_{k i}=1$ represents $k$ vehicle to meet customer $i$ demand; otherwise $y_{k i}=0$;

Then, the model of the vehicle routing problem as follows:

The objective function is:

$$
\min z=\sum_{i} \sum_{j} \sum_{k} c_{i j} \bullet x_{i j k}
$$

The constraint conditions are:

$$
\begin{array}{cc}
y_{k i} \in\{0,1\} & i \in \mathrm{m} ; \forall k \\
\sum_{k} y_{k i}=1 & i \in \mathrm{m} \\
\sum_{k \in \min \{k\}} i, j \in \mathrm{m} ; \forall k \\
\sum_{i} g_{i} y_{k i} \leq q & i \in \mathrm{m} ; \forall k \\
\sum_{i} x_{i j k}=y_{i k} & i \in \mathrm{m} ; \forall k \\
\sum_{j} x_{i j k}=y_{i k} & j \in \mathrm{m} ; \forall k \\
x_{i j} \in\{0,1\} & i \in \mathrm{m} ; \forall k
\end{array}
$$

The model is described as follows, formula (5) the minimum of total cost objective function; formula (6) as an integer constraint; formula (7) the demand of any customer satisfied only by a car; formula (8) distribution vehicle total cannot exceed the number of vehicles distribution center; the formula (9) the sum of the vehicle is responsible for any individual customer demand cannot exceed the maximum load of the vehicle; formula (10) and (11) express transportation route is a closed routes, after the vehicle logistics task is completed and return distribution center; formula (12) for integer constraints.

\section{THE SOlving STEPS}

$\mathrm{C}-\mathrm{W}$ saving algorithm thinking is simple, in solving the problem of the distribution route is a good algorithm, can quickly get a satisfactory solution to the problem. Through the algorithm will be improved, first shortest distance between each node with the shortest path method, and then use the saving algorithm, and finally thought insertion method, followed by the customer point insertion path to build distribution route [10-11]. The specific solving steps are as follows, the flow chart is shown Figure 2. 


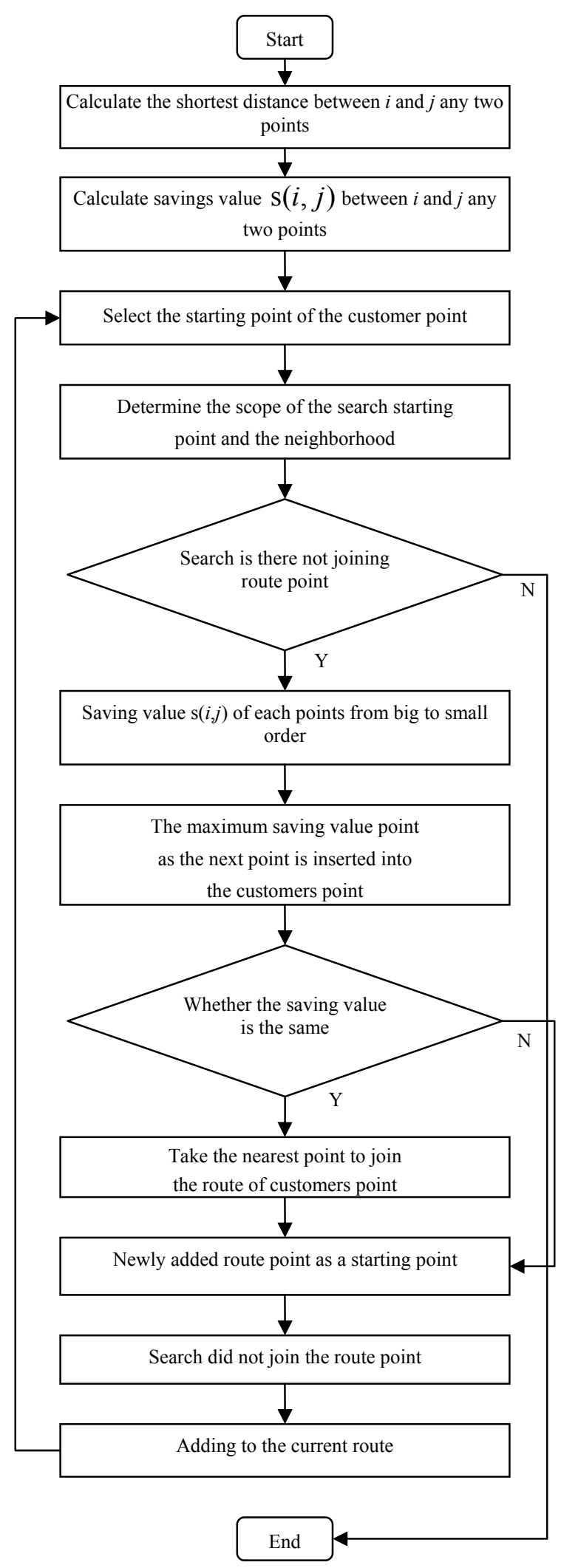

Figure 2. Flow chart
Use the shortest path method, to calculate the shortest distance between each node in the network.

The results of the above mentioned shortest path algorithm to calculate the savings mileage using c-w saving algorithm between any two points. According to the formula $s_{i, j}=d_{0, i}+d_{0, j}+d_{i, j}$ to calculate the value of saving $s_{i, j}$, so that the set $S=\{s(i, j) \mid s(i, j)>0\}$.

Using insertion method thinking, in turn the customer point insertion path to build the distribution route.

Selected distance from the customer point of furthest distribution center as a starting point;

Find the next point to be added to the route from the customer point of a client to determine the scope of the neighborhood around it, the aim is to reduce the search range, but also suitable for the requirements of the characteristics of the distribution. From this point of the set $S$ and other saving values $s_{i, j}$ in the neighborhood of each point removed, from big to small according to the order of ranking, take the point of maximum savings value is inserted as the next customer point; if have more than two savings values are equal, in the case, in accordance with the principles of the nearest neighbor method, whichever is nearest the customer point of adding the route;

To point the latest addition to the line as a starting point, in the vehicle capacity constraints and the maximum vehicle distribution restrictions on the number of users, repeat the steps to select and insert, when the expansion is no longer a path, then create another route until all customers are is discharged into the path.

\section{CONCLUSION}

The rapid development of modern society, and promote the continuous improvement of people's material and cultural life. Today, more and more fast-paced life, people in the busy work life apart, gradually became aware of the importance of physical fitness. Good health, physical fitness is a strong desire for everyone. The importance of exercise in today's society is not just because people simply like a sport, but because participation in these sports can be adjusted spirit, out of trouble, fatigue, prolong life, prevent and cure diseases and reduce health care costs, but also can improve the productivity of social labor, with positive implications for the development of the national economy. Therefore, people are ordering more and more fitness equipment, this paper optimized design fitness equipment distribution route.

Distribution lines optimization is always an important problem, effective distribution routes design, can save transportation mileage, saving time, thus reducing the most direct distribution costs, saving mileage method because of its simple, practical, applied more widely. Select a delivery two locations can shorten the distribution distance. If delivery to multiple users simultaneously, the saving algorithm according to save the distance size sequence planning of distribution line, it can greatly reduce transportation costs. As long as the vehicle is not overloaded, overrun, combined saving algorithm can reduce vehicle mileage, optimize the distribution line [12]. Based on the 
characteristics of saving algorithm, combined with the shortest path method and insertion method, the nearest neighbor method thinking was improve, when the distribution, there is a car loaded cargo of customers, designed along the best route in order to deliver goods to the customer, so can guarantee the goods to the customers' needs in a timely manner, but also save the vehicle, cost savings, ease the traffic tension pressure, and reduce the pollution caused by transport on the environment, scientific and rational design of fitness equipment and effective distribution route optimization to shorten transportation distances, reduce costs.

\section{ACKNOWLEDGMENT}

This work is supported by social science fund project of Liaoning province (L13BTY004).

\section{REFERENCES}

[1] Y. G. Le, "Study on Distribution Route Optimization in Traveling Salesman Problem Based on Saving Algorithm," Logistics Technology, vol. 33, no. 2, pp. 241-244, 2014.

[2] H. Li, "Beijing Tongyuan Logistics Enterprise Supply Chain Design and the Logistics Distribution Route Optimize," Northeastern University, 2007.

[3] K. J. Song, X. Z. Zhang, "Refined Oil Distribution Route Optimization Problem," Oil and Gas Storage and Transportation, vol. 27, no. 11, pp. 28-30, 2008.

[4] R. H. Sun, "Study of City Logistics Distribution System and its Routing Optimization Problem," Shandong University of Science and Technology, 2005.

[5] H. X. Liu, "The Analysis and Optimization of Logistics Distribution System of The Third Party Logistics Company," Hunan University, 2013.

[6] M. Liu, "Under the Environment of Supply Chain for the Research of Distribution Optimization Problem," Dalian Maritime University, 2013.

[7] E. J. Wu, "S Supermarket Distribution Center Location and Distribution Path Design," Nanjing University of Science and Technology, 2013.

[8] Q. L. Xv, "A Diagrammatizing Optimal Model of Vehicle Routing Based on GIS," Industrial Engineering and Management, vol. 12, no. 4, pp. 25-33, 2013.

[9] Y. H. Gao, "Optimization Research on Sales Logistics Distribution Path of The Liquor Enterprise," Jilin University, 2013.

[10] J. Gao, "The Research on Distribution Routing Problem Optimization for Jilin A Pharmaceutical Logistics Limited Company," Jilin University, 2014.

[11] Y. X. Liu, "Study on GIS Technology and Route Optimization Algorithm for Tobacco Logistics Distribution," Chongqing University, 2009

[12] K. Zhang, "The Study on Optimization of Logistics Distribution Paths in The Northwestern City," Xi'an University Of Architecture And Technology, 2011. 